\title{
Impact of Fluid Choice in Systemic Inflammatory Response Syndrome Patients on Hospital Cost Savings
}

\author{
Suzanne Laplante ${ }^{1} \cdot$ Dilip U. Makhija ${ }^{2} \cdot$ Sibyl H. Munson ${ }^{3} \cdot$ Victor S. Khangulov $^{3}$. \\ Fred W. Peyerl ${ }^{3}$ - Scott M. Paluszkiewicz ${ }^{3}$ - Aditi J. Ravindranath ${ }^{3}$. \\ Carol R. Schermer ${ }^{4}$
}

Published online: 4 September 2017

(C) The Author(s) 2017. This article is an open access publication

\begin{abstract}
Background There is growing evidence of the benefits of intravenous fluid therapy with balanced crystalloids over $0.9 \%$ 'normal' saline. This analysis evaluated the economic impact of increasing usage of a calcium-free balanced crystalloid solution (BAL) in patients with systemic inflammatory response syndrome (SIRS) on an annual hospital budget.

Methods An Excel ${ }^{\circledR}$-based economic model was developed to estimate costs associated with increased BAL usage (i.e., use in a greater proportion of patients), from the US hospital perspective, over a 5-year time horizon. Clinical inputs were based on the results of a retrospective Electronic Health Record (EHR) database analysis identifying significantly fewer complications among SIRS patients receiving predominantly BAL versus saline.
\end{abstract}

Electronic supplementary material The online version of this article (doi:10.1007/s41669-017-0055-y) contains supplementary material, which is available to authorized users.

Dilip U. Makhija

dilip_makhija@baxter.com

Suzanne Laplante

szlaplante@yahoo.fr

Sibyl H. Munson

sibyl.munson@bostonsp.com

Victor S. Khangulov

victor.khangulov@bostonsp.com

Fred W. Peyerl

fred.peyerl@bostonsp.com

Scott M. Paluszkiewicz

spalusz@gmail.com

Aditi J. Ravindranath

aditi.ravindranath@bostonsp.com
Complication-associated costs, adjusted to 2015, were obtained from published reports. Scenario analyses examined cost impacts for hospitals of various sizes, with different BAL adoption levels and rates.

Results Base-case scenario analysis (300-bed hospital, $80 \%$ occupancy, current and year 5 BAL usage in 5 and $75 \%$ of SIRS patients, respectively, exponential year-overyear adoption) showed year 1 hospital savings of US $\$ 29,232$ and cumulative 5-year savings of US $\$ 1.16 \mathrm{M}$. Cumulative 5-year pharmacy savings were US\$172,641. Scenario analyses demonstrated increasing cumulative 5 -year savings with increasing hospital size, year 5 BAL usage in greater proportions of patients, and rapid/early BAL adoption.

Conclusions Increased BAL usage represents an opportunity for hospitals and pharmacy departments to reduce complication-related costs associated with managing SIRS patients. The model suggests that savings could be expected across a range of scenarios, likely benefiting

Carol R. Schermer

cschermer@sbcglobal.net

1 Montreal, Canada

Baxter Healthcare Corporation, One Baxter Parkway, Deerfield, IL 60015, USA

3 Boston Strategic Partners, Inc., 4 Wellington Street, Suite 3, Boston, MA 02118, USA

4 Department of Surgery, Advocate Medical Group, Downers Grove, IL 60515, USA 
hospitals of various sizes and with different adoption capabilities.

\section{Key Points for Decision Makers}

The present economic analysis demonstrated that increased usage of calcium-free balanced crystalloid solution (BAL) in patients with systemic inflammatory response syndrome (SIRS) resulted in substantial cost savings for both the hospital and pharmacy in the US setting.

Savings were attributed to the reduction in key clinical complications such as sepsis, pneumonia, respiratory failure, and heart failure, which are significantly associated with crystalloid choice in SIRS patients. This is compelling considering the growing emphasis on healthcare cost containment with a focus on minimizing hospital adverse clinical outcomes.

Savings were observed outside of any additional reimbursement received for complications, and far outweighed the incremental fluid costs associated with increased BAL usage. Moreover, savings were evident across multiple scenarios, indicating potential economic benefits to hospitals of various sizes and varying adoption capability.

\section{Background}

Infusion of large volumes of intravenous (IV) fluids, with the goal of increasing intravascular volume and maintaining organ perfusion, is frequently employed in the management of critically ill patients or during surgery [1]. Patients managed with IV fluids include hypovolemic patients with systemic inflammatory response syndrome (SIRS), a condition frequently encountered in the hospitalized population, and in up to $\sim 20 \%$ of patients in the emergency department [2,3].

Several fluid types may be used for IV fluid therapy [1], and fluid selection can vary widely among physicians [2]. Crystalloid fluids differ considerably from each other with respect to their electrolyte content. For example, the most commonly used crystalloid, $0.9 \%$ 'normal' saline, contains supraphysiologic sodium and chloride concentrations (154 mmol/L), while 'balanced' fluids (e.g., Plasma-Lyte ${ }^{\circledR}$ [Baxter Healthcare, Deerfield, IL, USA], a calcium-free balanced fluid [BAL]) have electrolyte concentrations closer to the physiological range [1]. There is growing evidence of the important clinical implications of fluid choice [4-6]. Several studies have identified unfavorable clinical outcomes in acutely ill or surgical patients receiving $0.9 \%$ saline versus a balanced crystalloid [5, 7-10]. In addition, clinical complications attributed to fluid choice have been associated with greater healthcare resource usage in patients receiving $0.9 \%$ saline versus a balanced crystalloid [8-12]. In spite of this evidence, $0.9 \%$ saline remains widely used [13]. Broader usage of balanced crystalloids for IV therapy may in part be limited by higher acquisition costs when compared with $0.9 \%$ saline. For example, in the US BAL is priced $\sim 70 \%$ higher than saline [14]. However, a recent study demonstrated reduced $\mathrm{Mg}^{2+}$ requirements and a net $24-\mathrm{h}$ cost-benefit associated with resuscitation of trauma patients with a balanced crystalloid versus $0.9 \%$ saline [15].

A recent propensity-matched retrospective analysis of hospitalized patient data from a large US Electronic Health Records (EHR) database identified significantly reduced odds of serious adverse outcomes-such as sepsis, pneumonia, respiratory failure, heart failure, and mortality - in 3116 well matched patients with SIRS receiving IV fluid therapy with primarily BAL versus saline [16]. This reduction in serious adverse outcomes associated with fluid choice is expected to lead to a decrease in treatment-related costs, though no formal assessment of these savings has been carried out to date. The present analysis was conducted from a US hospital perspective to assess the impact of avoiding these IV fluid-related adverse outcomes in patients with SIRS on hospital cost savings.

\section{Methods}

\subsection{Perspective and Time Horizon}

An economic model was constructed in Microsoft Excel $2010^{\circledR}$ to assess the impact of increasing usage of BAL for IV fluid therapy in SIRS patients on the hospital budget (including the pharmacy budget). Monthly and yearly costs, as well as cumulative costs, were computed over a 5-year period.

\subsection{Patient Population and Interventions Compared}

The target population for the model was the same as that examined in the recent observational EHR database study [16]. A comprehensive review of PubMed, EMBASE, and the Cochrane CENTRAL databases performed through 2013 [5] and $>260$ additional publications published through October 12, 2016 yielded no additional relevant similar studies examining low- versus high-chloride 
solutions with similar attributes within SIRS patients. The observational study ([16]; NCT02083198) utilized the deidentified Health Insurance Portability and Accountability Act of 1996 (HIPAA)-compliant US database Health Facts (Cerner Corp., Kansas City, MO, USA), encompassing 486 clinical facilities, and which aggregates administrative data (International Classification of Diseases, Ninth Revision [ICD-9]) and clinical/quantitative data (medication and diagnostic test orders and laboratory results). A subset of inpatient data from January 1, 2009, through March 31, 2013, was utilized. The target population comprised adult (age $\geq 18$ years) hospitalized patients who met at least two SIRS criteria and received $\geq 500 \mathrm{~mL}$ of IV fluid within $48 \mathrm{~h}$ of first developing SIRS [16]. For this study, SIRS was defined as the presence of tachycardia (heart rate $>90 \mathrm{bpm}$ ) along with at least one of the following on the same day: (1) temperature $>38{ }^{\circ} \mathrm{C}$ or $<36{ }^{\circ} \mathrm{C}$, (2) respiratory rate $\geq 20$ breaths/minute or carbon dioxide partial pressure $\left(\mathrm{PaCO}_{2}\right) \leq 32 \mathrm{mmHg}$, or (3) leukocytes $\geq 12,000$ or $\leq 4000$ cells $/ \mathrm{mm}^{3}$. Exclusion criteria included receipt of $>1$ L of any IV fluid the day prior to SIRS qualification, receipt of any colloid or hypertonic saline, major cardiac surgeries, or a diagnosis of end-stage renal disease [16]. Propensity-matched cohorts were obtained from the unmatched cohort meeting inclusion/exclusion criteria, matching on patient and hospital demographics including patient age, gender, race, Elixhauser comorbidities, payer type, census region, bed size, admission source and type, urban versus rural setting, and teaching versus non-teaching hospital type [16, 17].

The primary outcome of the observational study was mortality $(3.27 \%$ saline [0.9\%] vs $1.03 \%$ BAL, $p<0.001)$, and secondary outcomes were length of stay (4.87 days saline vs 4.38 days $\mathrm{BAL}, \quad p=0.016)$, readmissions (13.54\% saline vs $10.91 \% \mathrm{BAL}$ at 60 days, $p=0.025)$, and complications defined through both administrative and clinical definitions (incorporating lab/microbiology results and medications) [16]. Outcomes were adjusted as described below. Findings from the EHR retrospective study revealed significant reductions in serious adverse outcomes in SIRS patients who received IV fluid therapy with BAL versus saline [16]. For example, the adjusted odds ratio and 95\% confidence intervals that were significantly in favor of BAL were mortality 0.378 (CI $0.211-0.676$ ), sepsis 0.568 (CI 0.431-0.75), pneumonia 0.393 (CI 0.259-0.596), respiratory failure 0.692 (CI $0.485-0.986$ ), and heart failure 0.401 (CI 0.246-0.653); while those that were significantly in favor of saline were low magnesium with replacement 0.546 (CI 0.355-0.84) and low potassium with replacement 0.551 (CI 0.429-0.706). SIRS frequency among hospitalized patients, stratified by hospital size (bed number; Table 1), was determined by calculating the mean monthly number of patients meeting SIRS criteria by hospital size in the EHR database and dividing by the average monthly patient volume for a given hospital size, as reported by the US Department of Health and Human Services [18].

The two interventions compared consisted of crystalloid therapy with and without BAL as the primary fluid. Both intervention cohorts comprised real-world patient populations who received a wide range of crystalloid therapies with a mix of fluid types and doses used. While all patients in the BAL group received at least $500 \mathrm{~mL}$ of BAL in addition to other commonly available crystalloids, patients in the saline group received at least $500 \mathrm{~mL}$ of $0.9 \%$ saline as their primary fluid along with other crystalloids, but did not receive any BAL. The fluid mix used in the model reflected that found in the observational study [16]. Patients in the BAL group received fluids in the following percentages, by volume: BAL $(55.3 \%) ; 0.9 \%$ saline $(25.8 \%)$; Ringer's Lactate $(17.2 \%)$; and $0.45 \%$ saline $(1.7 \%)$. For saline fluid therapy, patients received fluids in the following percentages, by volume: $0.9 \%$ saline $(76.3 \%)$; Ringer's Lactate $(21.3 \%) ; 0.45 \%$ saline $(2.4 \%)$.

\subsection{Model and Analytic Framework}

The impact on hospital savings of increasing usage of BAL in SIRS patients versus current BAL usage was computed yearly and over a 5-year period from the costs of IV fluids and fluid-related complications. For each level of BAL usage, which represents the proportion of SIRS patients receiving at least $500 \mathrm{~mL}$ BAL, the incremental frequency of clinical events including sepsis, pneumonia, cardiac dysrhythmias, cardiac stress (i.e., myocardial infarction/ acute coronary syndrome (MI/ACS), defined by abnormal troponin), heart failure, acute respiratory failure, and electrolyte replacement was calculated by interpolation of the observational study results [16] using the following equation:

$\mathrm{CF}_{\mathrm{BAL}_{i}}=\mathrm{CF}_{\text {Saline }}-\left[\left(\mathrm{CF}_{\text {Saline }}-\mathrm{CF}_{\mathrm{BAL}}\right) \times \mathrm{BAL}_{\text {usage }_{i}}\right]$

where $\mathrm{CF}_{\mathrm{BAL}_{i}}$ is the incremental complication frequency at BAL usage $i, \mathrm{CF}_{\text {Saline }}$ is the complication frequency among patients receiving crystalloid therapy with saline, $\mathrm{CF}_{\mathrm{BAL}}$ is the complication frequency among patients receiving crystalloid therapy with BAL, and $\mathrm{BALusage}_{i}$ is the proportion of SIRS patients receiving $\mathrm{BAL}$ as their primary IV fluid. It is important to note that $\mathrm{CF}_{\mathrm{BAL}_{i}}$ reflects the incremental frequency of clinical complications as patients move from saline to $\mathrm{BAL}$ at each stage of $\mathrm{BAL}$ usage $i$. Costs were calculated by multiplying complication frequency by per-episode treatment costs and the number of patients with SIRS. Annual fluid costs were determined by summation of fluid costs for the total annual number of SIRS patients. Annual savings were calculated by subtracting the costs of complications and treatments 
Table 1 Summary of model inputs

\begin{tabular}{|c|c|c|c|}
\hline Hospital bed size & \multicolumn{2}{|r|}{ Value $(\%)$} & Source \\
\hline \multicolumn{4}{|l|}{ SIRS frequency (\% of patients) } \\
\hline $0-99$ & \multicolumn{2}{|r|}{4.6} & Shaw et al. 2015 database outcomes analysis [16] \\
\hline $100-199$ & \multicolumn{2}{|r|}{4.7} & \\
\hline 200-299 & \multicolumn{2}{|r|}{3.8} & \\
\hline $300-499$ & \multicolumn{2}{|r|}{2.7} & \\
\hline$\geq 500$ & \multicolumn{2}{|r|}{1.8} & \\
\hline Average length of stay per patient & \multicolumn{2}{|r|}{4.62 days } & Shaw et al. 2015 database outcomes analysis [16] \\
\hline Complication & $\mathrm{BAL}^{\mathrm{a}}$ & Saline $^{\mathrm{b}}$ & Source \\
\hline \multicolumn{4}{|c|}{ Complication frequency ( $\%$ of SIRS patients; adjusted) } \\
\hline Death & 1.2 & 2.9 & Shaw et al. 2015 database outcomes analysis [16] \\
\hline 90-day readmission & 12.7 & 16.4 & \\
\hline Sepsis & 6.0 & 10.1 & \\
\hline Pneumonia & 2.3 & 5.7 & \\
\hline Cardiac dysrhythmia & 7.0 & 10.5 & \\
\hline Cardiac stress (MI/ACS) & 2.1 & 6.4 & \\
\hline Heart failure & 1.8 & 3.6 & \\
\hline Acute respiratory failure & 3.5 & 5.3 & \\
\hline $\mathrm{Mg}^{2+}$ replacement & 2.4 & 4.0 & \\
\hline $\mathrm{K}^{+}$replacement & 8.0 & 13.5 & \\
\hline
\end{tabular}

\begin{tabular}{|c|c|c|c|c|c|c|}
\hline \multicolumn{2}{|l|}{ Complication } & \multicolumn{2}{|l|}{ Cost (US\$) } & \multicolumn{3}{|c|}{ Source } \\
\hline \multicolumn{7}{|c|}{ Complication-associated cost $(\text { US\$/episode) })^{\mathrm{c}}$} \\
\hline Sepsis & & $12,955.87$ & \multicolumn{4}{|c|}{ Elixhauser et al., 2011 [20], Medicare Inpatient Charge Data, 2014 [23] } \\
\hline Pneumonia & \multicolumn{3}{|c|}{2456.67} & \multicolumn{3}{|c|}{ Pfuntner et al., 2013b [22], Medicare Inpatient Charge Data, 2014 [23] } \\
\hline Cardiac dysrhythmia & \multicolumn{3}{|c|}{2808.41} & \multicolumn{3}{|c|}{ Pfuntner et al., 2013a [21], Medicare Inpatient Charge Data, 2014 [23] } \\
\hline Cardiac stress (MI/ACS) & \multicolumn{3}{|c|}{$12,430.38$} & \multicolumn{3}{|c|}{ Pfuntner et al., 2013b [22], Medicare Inpatient Charge Data, 2014 [23] } \\
\hline Heart failure & \multicolumn{3}{|c|}{3931.84} & \multicolumn{3}{|c|}{ Pfuntner et al., 2013b [22], Medicare Inpatient Charge Data, 2014 [23] } \\
\hline Acute respiratory failure & \multicolumn{3}{|c|}{$16,769.62$} & \multicolumn{3}{|c|}{ Pfuntner et al., 2013a [21], Medicare Inpatient Charge Data, 2014 [23] } \\
\hline $\mathrm{Mg}^{2+}$ replacement & \multicolumn{2}{|r|}{13.56} & \multicolumn{4}{|c|}{ Shaw et al. 2015 database outcomes analysis [16] } \\
\hline $\mathrm{K}^{+}$replacement & \multicolumn{2}{|r|}{10.79} & \multicolumn{4}{|c|}{ Shaw et al. 2015 database outcomes analysis [16] } \\
\hline Complication & LOS & Medication & Procedure & Professional service & Diagnostic & Source \\
\hline \multicolumn{7}{|c|}{ Attributable costs (\% of total per-episode cost) } \\
\hline Sepsis & 33 & 20 & 6 & 17 & 24 & MS-DRG 2010 [24] \\
\hline Pneumonia & 32 & 18 & 6 & 19 & 25 & MS-DRG 2010 [24] \\
\hline Cardiac dysrhythmia & 36 & 13 & 5 & 21 & 24 & MS-DRG 2010 [24] \\
\hline Cardiac stress & 31 & 11 & 6 & 31 & 21 & MS-DRG 2010 [24] \\
\hline Heart failure & 37 & 11 & 5 & 21 & 26 & MS-DRG 2010 [24] \\
\hline Acute respiratory failure & 31 & 16 & 6 & 25 & 21 & MS-DRG 2010 [24] \\
\hline \multicolumn{3}{|l|}{ Fluid } & \multicolumn{2}{|c|}{ Cost } & \multicolumn{2}{|l|}{ Source } \\
\hline \multicolumn{7}{|l|}{ Per-liter fluid price (US\$) } \\
\hline \multicolumn{3}{|l|}{ BAL } & \multicolumn{2}{|c|}{11.46} & \multicolumn{2}{|c|}{ WAC, Micromedex Red Book, 2015 [14] } \\
\hline \multicolumn{3}{|l|}{ Ringer's Lactate } & \multicolumn{2}{|c|}{3.82} & \multicolumn{2}{|c|}{ WAC, Micromedex Red Book, 2015 [14] } \\
\hline \multicolumn{3}{|l|}{$0.9 \%$ saline } & \multicolumn{2}{|c|}{3.35} & \multicolumn{2}{|c|}{ WAC, Micromedex Red Book, 2015 [14] } \\
\hline
\end{tabular}


Table 1 continued

\begin{tabular}{lll}
\hline Fluid & Cost & Source \\
\hline $0.45 \%$ saline & 4.46 & WAC, Micromedex Red Book, 2015 [14] \\
\hline
\end{tabular}

$A C S$ acute coronary syndrome, BAL calcium-free balanced crystalloid solution, LOS length of stay, MI myocardial infarction, SIRS systemic inflammatory response syndrome, US\$ United States dollars, WAC wholesale acquisition cost

a BAL usage reflects use of a combination of crystalloids in the 3 days following the SIRS event, calculated from weighted average volumes (BAL: $54.5 \%$; $0.9 \%$ saline: $25.5 \%$; Ringer's Lactate: $17.0 \%$; $0.45 \%$ saline: $1.7 \%$ )

b Saline usage reflects use of a combination of crystalloids in the 3 days following the SIRS event, calculated from weighted average volumes (0.9\% saline: $76.0 \%$; Ringer's Lactate: $21.0 \%$; $0.45 \%$ saline: $2.0 \%$ )

b Per-episode complication costs were based on costs reported in the literature [20-22] offset by additional reimbursement hospitals receive for patients with major complications/co-morbidities [23]

associated with BAL adoption level for a given year from costs associated with current (year 0) BAL adoption level and subtracting the incremental costs of BAL. The following formula was used:

$\mathrm{AS}_{\mathrm{BAL}_{i}}=\left(\mathrm{CT}_{\mathrm{BAL}_{0}}-\mathrm{CT}_{\mathrm{BAL}_{i}}\right)-\mathrm{IC}_{\mathrm{BAL}_{i}}$

where $\mathrm{AS}_{\mathrm{BAL}_{i}}$ is the annual savings at $\mathrm{BAL}$ usage $i, \mathrm{CT}_{\mathrm{BAL}_{0}}$ is the cost of the treatment of complications associated with no BAL usage (current year 0 ), $\mathrm{CT}_{\mathrm{BAL}_{i}}$ is the cost of the treatment of complications associated with BAL usage $i$, and $\mathrm{IC}_{\mathrm{BAL}_{i}}$ is the incremental costs of BAL at BAL usage $i$.

\subsection{Model Inputs and Data Sources}

Model inputs, including SIRS frequency and complication frequencies in patients meeting SIRS criteria (Table 1), were based on clinical outcome rates, adjusted for acute physiology score (APS) from the APACHE II classification system [19], and patient and hospital variables that differed between cohorts after propensity matching ( $p<0.10$ ), as reported in the observational study [16]. SIRS frequencies stratified by hospital bed size (Table 1) were determined from the average number of SIRS patients per month and total number of patients per month for each hospital bed-size level, derived from the Cerner Health Facts database outcomes analysis [16]. Average length of stay per patient was also identified from the Cerner Health Facts database [16].

Only outcome parameters that were significantly different, after outcomes adjustments, were utilized as economic outcomes within the model. Due to less than a quarter of patients' records containing charge data within the database, published US sources for costs were obtained. Sources were identified via web engine searches and the PubMed database. Search terms used in PubMed included complication names (e.g., pneumonia, heart failure) and the MeSH heading 'economics'.

Per-episode complication-associated costs (Table 1) were obtained from Healthcare Utilization Project (HCUP) statistical briefs published by the US Agency for Healthcare Research and Quality (AHRQ) [20-22], which provides the inflation-adjusted mean cost per stay for a principal diagnosis of a given complication. Costs were determined from total hospital charges through application of an HCUP costto-charge ratio derived from accounting reports for each individual hospital from Centers for Medicare and Medicaid Services (CMS) [19-21]. In order to more accurately reflect true costs to hospitals for treatment of complications, additional reimbursement hospitals may receive for patients with major complications was deducted from the per-episode complication-associated costs. The reimbursement offset amount was calculated as representative of 7 million discharges or $75 \%$ of Medicare Inpatient Prospective Payment System (IPPS) discharges in the 2014 financial year, by using the average difference across Diagnosis-Related Group (DRG) code pairs without versus with major complications/co-morbidities (MCC) and weighted based on number of discharges for a given DRG code pair, as reported in Medicare Inpatient Charge Data [23].

Attributable costs (respective fractions of per-episode cost attributable to length of stay [LOS], medications, procedures performed, professional services, and diagnostic procedures) were obtained from published reports and the MS-DRG Handbook [24]. The attributable cost fraction, when obtained from the Handbook, was based on the proportional breakdown of average charge per discharge reported in the MedPAR data source released annually by CMS. Based on the appropriate DRG determined for a clinical event of interest from the EHR database, proportional cost for cost channels provided in the Handbook were converted to cost channels identified in the model (MS-DRG Handbook cost channels were included within the model according to the following: General care and Special care (Length-of-stay within the model), Pharmacy (Medication), Operating room and Medical supplies (Procedure), Anesthesiology and other ancillary (Professional services), and Laboratory and Radiology (Diagnostics).

Per-liter fluid costs were defined as the wholesale acquisition cost (WAC) in US dollars (USD), obtained from the Micromedex Red Book (October 2015) [14]. Electrolyte replacement costs were estimated based on the 
weighted average hospital cost for all products used in the observational study dataset. Mean 72-hour post-SIRS qualification IV fluid volumes used to manage all SIRS patients were obtained from the database [16]. All cost inputs were adjusted to 2015 USD using the consumer price index [25].

\subsection{Analyses and Outputs}

The base case was defined as a 300-bed hospital with $80 \%$ occupancy, a $2.7 \%$ SIRS frequency in the hospital patient population [16] (42 SIRS patients requiring IV fluid therapy/month, using 4.62 days average length of stay per patient [16]), current BAL usage in 5\% of SIRS patients, projected year 5 BAL usage in $75 \%$ of SIRS patients, and exponential year-over-year BAL adoption (Supplementary Table 1, see electronic supplementary material [ESM]). Complication frequencies and costs are reported for current BAL usage and for each subsequent year. Annual savings were computed as the difference between cost for a given year and current cost, and cumulated over the 5-year period. Hospital-level costs include costs attributable to LOS, medications, procedures performed, professional services, diagnostic procedures, and 72-h fluid usage. Pharmacylevel costs include costs attributable to medications and 72-h fluid usage. All figures were created with Microsoft Excel $2010^{\circledR}$.

\subsection{Sensitivity and Scenario Analysis}

One-way and probabilistic sensitivity analyses were carried out to account for uncertainty around estimates for model input parameters, which included the number of SIRS patients requiring IV fluid therapy, per-episode complication costs, per-liter fluid costs, and differences in individual complication frequencies between patients receiving BAL or saline. One-way sensitivity analysis was performed by individually varying model parameters. Per-episode complication costs were independently varied by $\pm 75 \%$. All other parameters were varied by $\pm 20 \%$ (Supplementary Table 2, see ESM). In contrast to the one-way sensitivity analysis, the probabilistic sensitivity analysis was performed with Monte Carlo simulation where all inputs were varied simultaneously. One thousand iterations were run and convergence monitoring was carried out to ensure sufficient number of iterations were performed. The Normal distribution with mean and standard deviation as specified in Supplementary Table 3 (see ESM) was assumed for all model parameters. Standard deviation was estimated using published approximations [26]. Probabilistic sensitivity analysis was performed using @RISK software (PalisadeCorp., Ithaca, NY, USA).
Scenario analyses were performed to account for the uncertainty around the model structure and included examining the impact of different hospital sizes $(100,300$, or 600 beds), different target year 5 BAL usages ( 25 or 95\%), and different year-over-year adoption strategies (exponential or linear) on cumulative 5-year savings (Supplementary Table 1, see ESM).

\section{Results}

\subsection{Clinical and Economic Outcomes: Base Case}

Increasing BAL usage from $5 \%$ to $75 \%$ of SIRS patients resulted in a reduction in complication rates, 90-day readmission rates, and electrolyte replacement (Table 2). The decrease in complications due to increased BAL usage led to cost savings compared with current usage as early as year 1 (9\% BAL usage). For the base case, cumulative savings versus current usage were estimated to be US $\$ 29,232$ by year 1 and US $\$ 1.16 \mathrm{M}$ by year 5 (75\% BAL usage). These savings were evident despite increased fluid costs, from a current cost of US\$5312, to US\$9284 by year 5. The largest contributors to savings were LOS, professional services, and diagnostic costs. At the pharmacy level, savings were estimated to be US $\$ 4375$ in the first year and total cumulative savings were US\$172,641 over the 5-year period (Table 2).

\subsection{Sensitivity and Scenario Analysis}

Probabilistic sensitivity analysis showed that for 5-year cumulative savings, the $95 \%$ CI was between US $\$ 1.15$ and US\$1.18 M, with a mean of US\$1.16 M and SD of US $\$ 254,177$. The parameters with the largest impact on savings were the costs associated with managing episodes of sepsis and cardiac stress (MI/ACS), as well as the number of patients with SIRS requiring IV fluid therapy (Fig. 1). This was also in agreement with results from the one-way sensitivity analysis (Supplementary Table 4, see ESM). When the cost of managing an episode of sepsis or cardiac stress was varied, 5-year cumulative savings ranged between US $\$ 0.87 \mathrm{M}$ and US $\$ 1.40 \mathrm{M}$ and US $\$ 0.93 \mathrm{M}$ and US $\$ 1.38 \mathrm{M}$, respectively (versus US $\$ 1.16 \mathrm{M}$ in the base case). Similarly, cumulative savings ranged between US\$0.98 M and US\$1.35 M when the number of SIRS patients requiring IV fluid therapy per month was varied. With respect to complication frequencies, changes in the frequency of acute respiratory failure, cardiac stress, and sepsis had the greatest impacts on 5-year cumulative savings, whereas changes in cumulative savings associated with increases or decreases in electrolyte replacement costs and per-liter fluid costs were relatively small. None of the 
Table 2 Clinical and economic outcomes associated with increased BAL usage, base case

\begin{tabular}{|c|c|c|c|c|c|c|}
\hline & \multicolumn{6}{|c|}{ Year (\% BAL usage) } \\
\hline & Current $(5 \%)$ & Year $1(9 \%)$ & Year $2(15 \%)$ & Year $3(25 \%)$ & Year $4(44 \%)$ & Year $5(75 \%)$ \\
\hline \multicolumn{7}{|c|}{ Complications, $\%$ of SIRS patients $\left(n /\right.$ month $\left.^{\mathrm{a}}\right)$} \\
\hline Death & $2.8(1)$ & $2.8(1)$ & $2.6(1)$ & $2.5(1)$ & $2.2(1)$ & $1.6(1)$ \\
\hline 90-day readmission & $16.2(7)$ & $16.1(7)$ & $15.9(7)$ & $15.5(6)$ & $14.8(6)$ & $13.6(6)$ \\
\hline Sepsis & $9.9(4)$ & $9.7(4)$ & $9.5(4)$ & $9.0(4)$ & $8.3(3)$ & $7.0(3)$ \\
\hline Pneumonia & $5.5(2)$ & $5.4(2)$ & $5.2(2)$ & $4.8(2)$ & $4.2(2)$ & $3.1(1)$ \\
\hline Cardiac dysrhythmia & $10.3(4)$ & $10.2(4)$ & $10.0(4)$ & $9.6(4)$ & $9.0(4)$ & $7.9(3)$ \\
\hline Cardiac stress (MI/ACS) & $6.1(3)$ & $6.0(3)$ & $5.7(2)$ & $5.3(2)$ & $4.5(2)$ & $3.1(1)$ \\
\hline Heart failure & $3.5(1)$ & $3.4(1)$ & $3.3(1)$ & $3.1(1)$ & $2.8(1)$ & $2.2(1)$ \\
\hline Acute respiratory failure & $5.3(2)$ & $5.2(2)$ & $5.1(2)$ & $4.9(2)$ & $4.6(2)$ & $4.0(2)$ \\
\hline $\mathrm{Mg}^{2+}$ replacement & $3.9(2)$ & $3.9(2)$ & $3.8(2)$ & $3.6(2)$ & $3.3(1)$ & $2.8(1)$ \\
\hline $\mathrm{K}^{+}$replacement & $13.2(6)$ & $13.0(5)$ & $12.6(5)$ & $12.1(5)$ & $11.1(5)$ & $9.3(4)$ \\
\hline \multicolumn{7}{|l|}{ Economic outcomes (US\$) } \\
\hline \multicolumn{7}{|l|}{ Attributable cost } \\
\hline LOS & 568,123 & 558,669 & 542,419 & 514,490 & 466,487 & 383,979 \\
\hline Medication & 279,718 & 275,139 & 267,275 & 253,778 & 230,639 & 191,039 \\
\hline Procedure & 105,414 & 103,673 & 100,680 & 95,537 & 86,696 & 71,501 \\
\hline Professional services & 399,897 & 392,940 & 380,983 & 360,432 & 325,108 & 264,396 \\
\hline Diagnostic & 402,524 & 395,819 & 384,296 & 364,489 & 330,447 & 271,935 \\
\hline 3-day (72 h) fluid cost & 5312 & 5516 & 5866 & 6468 & 7504 & 9284 \\
\hline \multicolumn{7}{|l|}{ Total—hospital $^{\text {b }}$} \\
\hline Total annual cost & $1,760,987$ & $1,731,755$ & $1,681,519$ & $1,595,194$ & $1,446,880$ & $1,192,135$ \\
\hline Total annual savings & 0 & 29,232 & 79,468 & 165,793 & 314,107 & 568,852 \\
\hline Total cumulative savings & & 29,232 & 108,700 & 274,492 & 588,599 & $1,157,452$ \\
\hline \multicolumn{7}{|l|}{ Total—pharmacy ${ }^{\mathrm{c}}$} \\
\hline Total annual cost & 285,030 & 280,654 & 273,141 & 260,247 & 238,142 & 200,323 \\
\hline Total annual savings & 0 & 4375 & 11,889 & 24,783 & 46,887 & 84,707 \\
\hline Total cumulative savings & & 4375 & 16,264 & 41,048 & 87,935 & 172,641 \\
\hline
\end{tabular}

$A C S$ acute coronary syndrome, BAL calcium-free balanced crystalloid solution, LOS length of stay, MI myocardial infarction, SIRS systemic inflammatory response syndrome, US\$ United States dollars

a $n /$ month rounded to nearest unit

b Includes LOS, medication, procedure, professional services, diagnostic, and 3-day fluid costs

c Includes medication and 3-day fluid costs

variables evaluated in the sensitivity analysis resulted in increased costs over the current usage of BAL.

Keeping all other base-case parameters constant, analysis of mortality showed that BAL usage in an increasing number of patients resulted in decreasing SIRS patient mortality, particularly for years 3-5 (Fig. 2). For a hospital using BAL in $95 \%$ of SIRS patients by year 5 , mortality among SIRS patients decreased from 2.8 to $1.3 \%$ in year 5 . Even with more modest BAL adoption ( $25 \%$ by year 5 ), mortality was found to decrease to $2.5 \%$ (Fig. 2a). These reductions in mortality rate translated to around eight fewer annual SIRS patient deaths in year 5 for a 300-bed hospital using BAL for $95 \%$ of SIRS patients and around two fewer annual SIRS patient deaths in year 5 with $25 \%$ BAL adoption (Fig. 2b).
In order to assess potential savings across a range of situations, a series of scenarios was tested (Fig. 3) by varying hospital size $(100,300$, and 600 beds), year 5 BAL adoption level (use in $95 \%$ and $25 \%$ of SIRS patients), and rate of adoption (exponential and linear year-over-year increases in BAL usage). The model estimated that cumulative savings increased with hospital size (i.e., number of patients with SIRS) and year 5 BAL usage level, with savings as high as US\$1.96 M for a 600-bed hospital with 95\% year 5 BAL usage and exponential adoption (Fig. 3a). For the smallest hospital size examined (100 beds) and more modest (25\%) projected year 5 BAL usage, cumulative savings were estimated to be US\$221,530. Finally, rapid/early (linear year-over-year) adoption was associated with greater cumulative savings than 


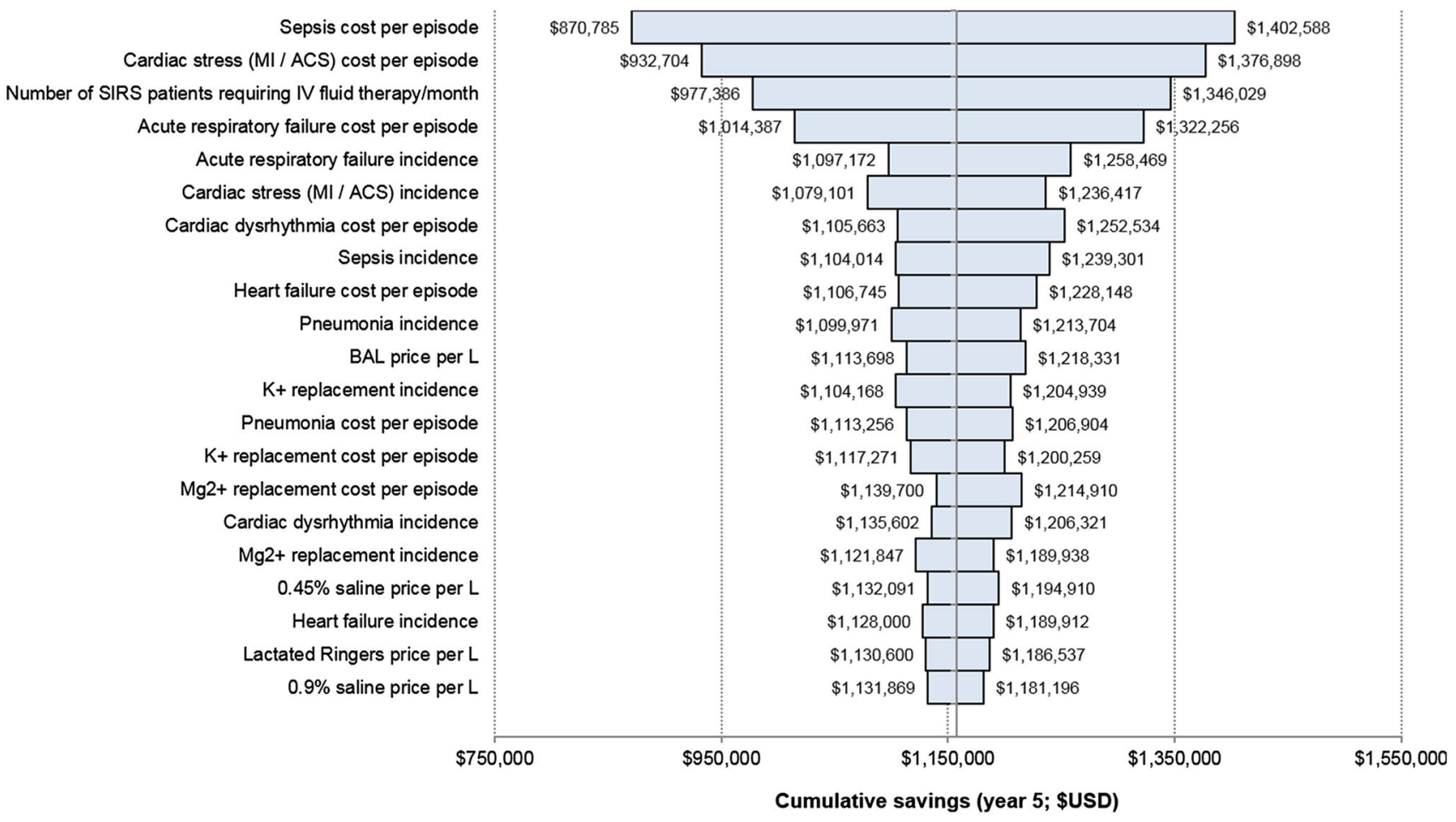

Fig. 1 Probabilistic sensitivity analysis of cumulative 5-year savings. This figure presents the results of the user inputs of the probabilistic sensitivity analysis. All parameters were assumed to be Normally distributed and varied simultaneously with means and standard deviations as specified in Supplementary Table 3 (see ESM). Bars represent maximum and minimum cumulative savings associated with

exponential year-over-year increases. For a 300-bed hospital with a target year 5 BAL usage in $95 \%$ of SIRS patients, cumulative 5-year savings reached US $\$ 2.19 \mathrm{M}$ with rapid/early adoption versus US $\$ 1.44 \mathrm{M}$ with exponential adoption over 5 years. As in the base case, savings in the scenario analyses were driven by reductions in complications. Annual reductions in complication rates were greater with increasing hospital size and BAL adoption (Fig. 3b).

\section{Discussion}

Growing emphasis on cost containment in healthcare means that hospitals must develop strategies to minimize adverse clinical outcomes while increasing cost efficiency. The present analysis from a hospital perspective demonstrates that using BAL in an increasing percentage of SIRS patients could result in substantial cost savings for the hospital and pharmacy. These savings are expected to result from lower numbers of key clinical complications that are significantly associated with crystalloid choice in patients with SIRS [16]. The model demonstrates that savings can be realized as soon as year 1 , despite increased fluid costs. Savings changes in the model input values. The midline represents the mean cumulative savings at US\$1.16M, with $95 \%$ CI between US\$1.15 and US\$1.18 M. Inputs are ranked by effect on cumulative 5-year savings. BAL calcium-free balanced crystalloid solution, MI/ACS myocardial infarction/acute coronary syndrome, SIRS systemic inflammatory response syndrome

were evident across the scenarios that were tested, suggesting potential economic benefits to hospitals of various sizes and with different adoption capabilities.

In addition to the hospital and its associated pharmacy, the reduced complication rates seen with increased BAL utilization among SIRS patients could indirectly impact other healthcare providers, including outpatient care and rehabilitation services that could potentially treat patients recovering from SIRS-associated complications.

This analysis has some limitations, one of which is the use of clinical efficacy results from a single observational study [16] as central model inputs, due to lack of other relevant studies after a comprehensive literature search ([5] and above). This observational study examined data from a large US EHR database containing patient data from hospitals nationwide. Thus, the clinical inputs represent realworld outcomes across hospitals of various sizes and specialties serving diverse patient populations. Furthermore, the use of a single set of inclusion criteria and propensitymatched cohorts in that study provides the advantage of a well defined and relatively uniform patient population. In addition, this observational study [16] is one of many recent studies that support and expand the growing evidence regarding the potential risks associated with $0.9 \%$ 

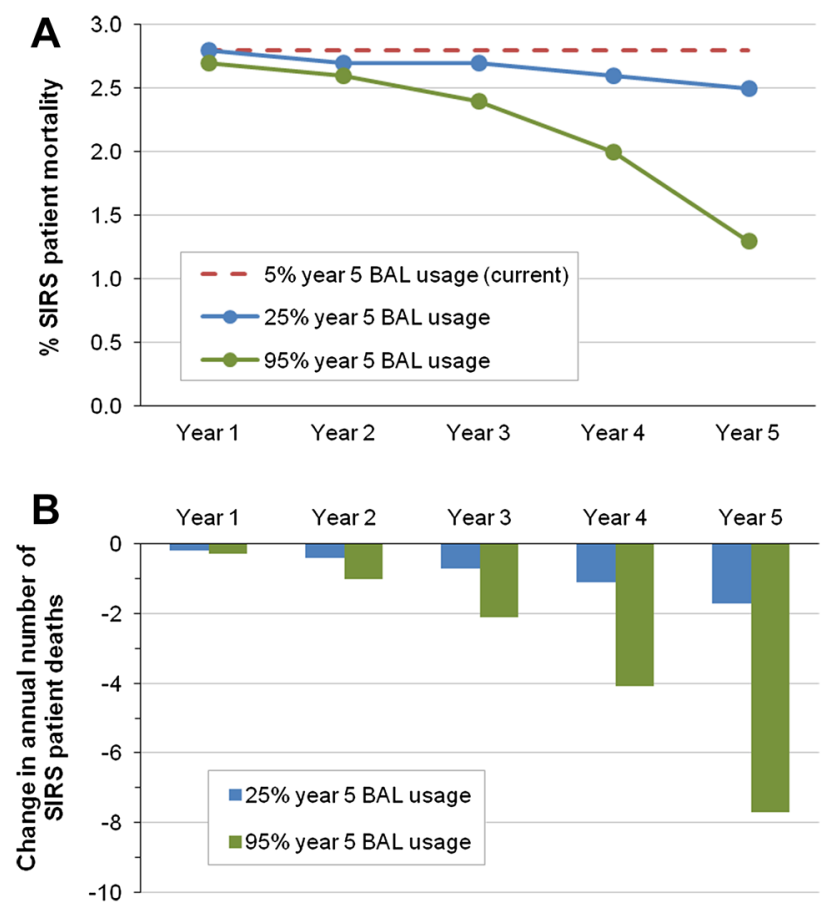

Fig. 2 Mortality scenario analyses. Annual SIRS patient mortality rate (a) and change in the number of systemic inflammatory response syndrome $(S I R S)$ patient deaths (b), years 1-5, for different year 5 calcium-free balanced crystalloid solution $(B A L)$ usage scenarios. Dotted line in a represents annual mortality rate assuming no change in BAL usage from baseline, as defined in the base case (5\% usage)

saline. Several prospective and retrospective studies have identified lower adverse outcome rates [7-10] and healthcare resource usage, including reduced fluid, blood transfusion, and mechanical ventilation requirements [8-12] in patients receiving balanced crystalloids versus $0.9 \%$ saline. We therefore expect that the savings estimated in the current analysis would likely be observed with a different set of data derived from similar observational studies or meta-analyses.

Comparison of the two arms of the EHR retrospective study [16] may also present limitations with possible differences in active physician management of patients in each cohort representing a source of bias. However, to reduce chance of bias, extensive propensity matching was carried out using multiple hospital characteristics, patient demographics, and surgical procedures.

In addition to the clinical inputs, the cost components also represent limitations to this analysis. Complication cost inputs were obtained from the medical literature reporting studies using large national databases, and thus are likely to provide reliable estimates; however, these costs can change over time, which could have an impact on savings. Due to the lack of appropriate sources for costs associated with treating multiple complications in hospitalized patients (which may vary based on each patient's unique condition), and a SIRS population specifically, complication cost data was modeled with the underlying assumption that for a single patient the costs would be additive. However, the one-way sensitivity analysis took into account minimum costs that would be in line with low complication treatment costs. It is also worth noting that this assumption was applied to both cohorts, impacting both to a similar extent, and hence may have a minimal impact on the cost-savings output.
Fig. 3 Cumulative savings and complications scenario analyses. a Cumulative cost difference (years 1-5) for different hospital size and calcium-free balanced crystalloid solution $(B A L)$ adoption scenarios. Greatest cumulative savings (i.e., largest cumulative cost differences) are associated with larger hospital size, greater year 5 BAL usage, and rapid/early (linear) adoption. b Annual change in overall complication number for different hospital size and BAL adoption scenarios. Largest reductions in complications are associated with larger hospital size and greater BAL usage
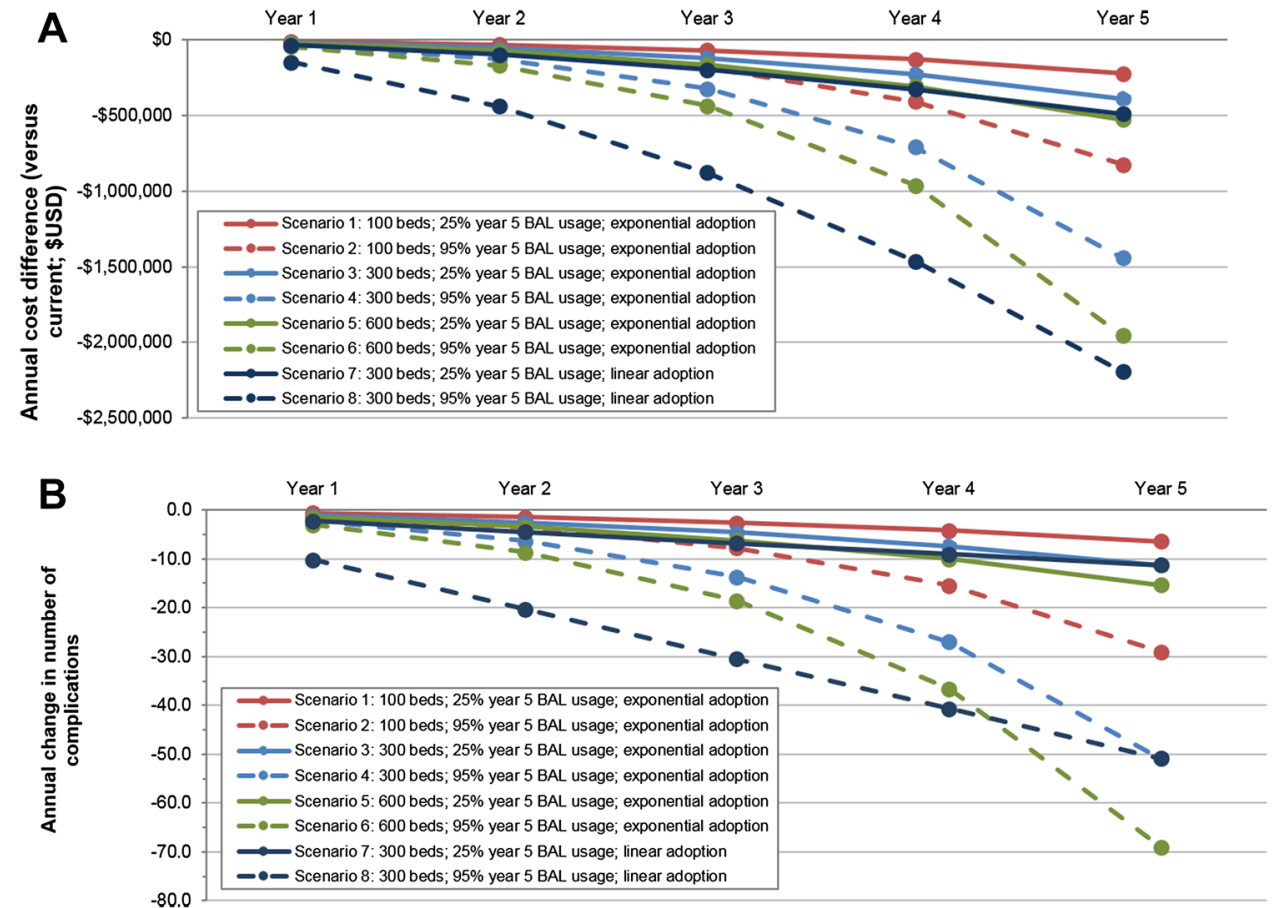
Another limitation is that hospital readmissions were measured and reported solely as clinical outcomes in this study, and are not included in the economic and sensitivity analysis. However, it is worth noting that readmissions could have an economic impact, particularly when a hospital reimbursement penalty results under the CMS Hospital Readmission Reduction Program (relevant to patients with acute myocardial infarction, heart failure, pneumonia, chronic obstructive pulmonary disease, elective total hip arthroplasty, and total knee arthroplasty) [27].

Different scenarios representing a range of plausible institutional considerations were performed. However, the results of this analysis may not be generalizable to institutions in other countries. While the clinical evidence from the database analysis is likely to be transferable to analyses for hospitals in other countries, it should be noted that specific clinical practices for the management of complications, and therefore costs, may differ from those used in the model, and thus costs would need to be customized to other countries. In addition, the outcomes analysis used to develop the model investigated a heterogeneous, matched patient population; however, the relative impact of BAL adoption in different at-risk patient groups, such as the elderly, requires further evaluation. Finally, it is important to note that this analysis examined a population of patients meeting SIRS criteria, and that the cost impact of increased BAL usage for non-SIRS patients remains to be evaluated.

Crystalloid choice represents a readily modifiable component of clinical practice. Given that (1) IV fluid therapy is a frequently employed practice and (2) $0.9 \%$ saline continues to be widely used, many hospitals and pharmacy departments may benefit from a shift to greater use of balanced crystalloids for patients meeting SIRS criteria. While the use of alternatives to $0.9 \%$ saline may currently be limited due to concerns regarding fluid costs, the present study clearly demonstrates the potential for hospital- and pharmacy-level cost savings associated with reducing the incidence of key clinical complications in SIRS patients. As expected, the model estimates increased fluid costs with greater BAL usage (base case defined a BAL cost threefold greater than $0.9 \%$ saline cost); however, these costs are greatly outweighed by the savings associated with reduced complication rates and medication usage to treat these complications.

\section{Conclusion}

The present economic analysis, based on the results of realworld clinical outcomes analysis and using published US cost data, suggests that usage of a calcium-free balanced crystalloid for IV fluid therapy in an increasing number of patients with SIRS could generate sizeable savings. Base- case scenario analysis (300-bed hospital, $80 \%$ occupancy, current and year 5 BAL usage in 5 and $75 \%$ of SIRS patients, respectively, exponential year-over-year adoption) showed cumulative 5-year savings of US\$1.16 M for hospitals, with US\$172,641 in cumulative savings specifically for pharmacy departments.

Author contributions S Laplante was responsible for concept and design, and analysis and interpretation of data. DU Makhija was responsible for analysis and interpretation of data, drafting and critical revision of the manuscript. SH Munson was responsible for concept and design, acquisition, analysis and interpretation of data, and critical revision of the manuscript. VS Khangulov was responsible for concept and design, acquisition, analysis and interpretation of data, drafting and critical revision of the manuscript, and statistical analysis. FW Peyerl was responsible for concept and design, and analysis and interpretation of data. SM Paluszkiewicz was responsible for acquisition, analysis and interpretation of data, and drafting and critical revision of the manuscript. AJ Ravindranath was responsible for acquisition, analysis and interpretation of data, and critical revision of the manuscript. CR Schermer was responsible for concept and design, analysis and interpretation of data, and drafting and critical revision of the manuscript.

\section{Compliance with Ethical Standards}

Ethics approval and consent to participate Not applicable.

Consent for publication Not applicable.

Availability of data and materials All data generated or analyzed during this study are included in this published article (and its supplementary information files).

Conflicts of interest DU Makhija is an employee of Baxter Healthcare Corporation. S Laplante and CR Schermer were employees of Baxter Healthcare Corporation at the time of the study. SH Munson, VS Khangulov, FW Peyerl, and AJ Ravindranath are consultants at Boston Strategic Partners, Inc., funded by Baxter Healthcare Corporation to conduct the study analyses. SM Paluszkiewicz was a consultant at Boston Strategic Partners, Inc. during the time of the study. This study was funded by Baxter Healthcare Corporation, Deerfield, IL, USA.

Funding This study was funded by Baxter Healthcare Corporation, Deerfield, IL, USA.

Open Access This article is distributed under the terms of the Creative Commons Attribution 4.0 International License (http:// creativecommons.org/licenses/by/4.0/), which permits unrestricted use, distribution, and reproduction in any medium, provided you give appropriate credit to the original author(s) and the source, provide a link to the Creative Commons license, and indicate if changes were made.

\section{References}

1. Lobo DN, Lewington AJ, Allison SP. Basic concepts of fluid and electrolyte therapy. Melsungen: Bibliomed; 2013.

2. Finfer S, Liu B, Taylor C, Bellomo R, Billot L, Cook D, et al. Resuscitation fluid use in critically ill adults: an international 
cross-sectional study in 391 intensive care units. Crit Care. 2010;14(5):R185. doi:10.1186/cc9293.

3. Horeczko T, Green JP, Panacek EA. Epidemiology of the Systemic Inflammatory Response Syndrome (SIRS) in the Emergency Department. West J Emerg Med. 2014;15(3):329-36.

4. Guidet B, Soni N, Della Rocca G, Kozek S, Vallet B, Annane D, et al. A balanced view of balanced solutions. Crit Care. 2010;14(5):325.

5. Krajewski ML, Raghunathan K, Paluszkiewicz SM, Schermer $\mathrm{CR}$, Shaw AD. Meta-analysis of high- versus low-chloride content in perioperative and critical care fluid resuscitation. $\mathrm{Br} \mathrm{J}$ Surg. 2015;102(1):24-36.

6. Lobo DN. Intravenous $0.9 \%$ saline and general surgical patients: a problem, not a solution. Ann Surg. 2012;255(5):830-2.

7. Raghunathan K, Shaw AD, Nathanson B, Stürmer T, Brookhart A, Stefan MS, et al. Association between the choice of IV crystalloid and in-hospital mortality among critically ill adults with sepsis. Crit Care Med. 2014;42(7):1585-91.

8. Shaw AD, Bagshaw SM, Goldstein SL, Scherer LA, Duan M, Schermer CR, et al. Major complications, mortality, and resource utilization after open abdominal surgery: $0.9 \%$ saline compared to Plasma-Lyte. Ann Surg. 2012;255(5):821-9. doi:10.1097/SLA. 0b013e31825074f5.

9. Waters JH, Gottlieb A, Schoenwald P, Popovich MJ, Sprung J, Nelson DR. Normal saline versus lactated Ringer's solution for intraoperative fluid management in patients undergoing abdominal aortic aneurysm repair: an outcome study. Anesth Analg. 2001;93(4):817-22.

10. Young JB, Utter GH, Schermer CR, Galante JM, Phan HH, Yang $\mathrm{Y}$, et al. Saline versus plasma-lyte A in initial resuscitation of trauma patients: a randomized trial. Ann Surg. 2013;259(2):255-62. doi:10.1097/SLA.0b013e318295feba.

11. Chua HR, Venkatesh B, Stachowski E, Schneider AG, Perkins K, Ladanyi S, et al. Plasma-Lyte 148 vs $0.9 \%$ saline for fluid resuscitation in diabetic ketoacidosis. $\mathrm{J}$ Crit Care. 2012;27(2):138-45. doi:10.1016/j.jcrc.2012.01.007.

12. Mahajan V, Sajan SS, Sharma A, Kaur J. Ringers lactate vs Normal saline for children with acute diarrhea and severe dehydration- a double blind randomized controlled trial. Indian Pediatr. 2012;49(12):963-8. doi:10.1007/s13312-012-0251-x.

13. Wan L, Bellomo R, May CN. The effects of normal and hypertonic saline on regional blood flow and oxygen delivery. Anesth Analg. 2007;105(1):141-7.

14. Red Book. Thomson Reuters Micromedex Clinical Evidence Solutions [online database] http://thomsonreuters.com/products_ services/healthcare/healthcare_products/clinical_deci_support/ micromedex_clinical_evidence_sols/med_safety_solutions/red_ book/. Accessed Oct 2015.

15. Smith CA, Duby JJ, Utter GH, Galante JM, Scherer LA, Schermer CR. Cost-minimization analysis of two fluid products for resuscitation of critically injured trauma patients. Am J Health Syst Pharm. 2014;71:470-5.

16. Shaw AD, Schermer CR, Lobo DN, Munson SH, Khangulov V, Hayashida D et al. Impact of intravenous fluid composition on outcomes in patients with the systemic inflammatory response syndrome. Critical Care (London, England). 2015;19(1):334.

17. Elixhauser A, Steiner C, Harris DR, Coffey RM. Comorbidity measures for use with administrative data. Med Care. 1998;36(1):8-27.

18. National Center for Health Statistics. Health, United States, 2011: With Special Feature on Socioeconomic Status and Health. Hyattsville, MD: US Department of Health and Human Services2012 May. Report No.: DHHS report no. 2012-1232.

19. Knaus WA, Draper EA, Wagner DP, Zimmerman JE. APACHE II: a severity of disease classification system. Crit Care Med. 1985;18(10):818-29.

20. Elixhauser A, Friedman B, Stranges E. Septicemia in U.S. Hospitals, 2009. HCUP Statistical Brief \#122. Rockville: Agency for Healthcare Research and Quality; 2011.

21. Pfuntner A, Wier LM, Steiner C. Costs for Hospital Stays in the United States, 2010. HCUP Statistical Brief \#146. Rockville: Agency for Healthcare Research and Quality; 2013.

22. Pfuntner A, Wier LM, Steiner C. Costs for Hospital Stays in the United States, 2011. HCUP Statistical Brief \#168. Rockville: Agency for Healthcare Research and Quality; 2013.

23. National and State Summaries of Inpatient Charge Data FY2014. Centers for Medicare and Medicaid Services. 2014. https://www. cms.gov/Research-Statistics-Data-and-Systems/Statistics-Trendsand-Reports/Medicare-Provider-Charge-Data/Inpatient2014. html. Accessed June 2017.

24. Thomson Reuters. MS-DRG handbook: comparative clinical and financial benchmarks. 2010.

25. United States Bureau of Labor and Statistics [online database]. CPI Inflation Calculator. http://www.bls.gov/data/inflation calculator.htm. Accessed Oct 2015.

26. Hozo SP, Djulbegovic B, Hozo I. Estimating the mean and variance from the median, range, and the size of a sample. BMC Med Res Methodol. 2005;5:13. doi:10.1186/1471-2288-5-13.

27. Fingar K, Washington R. Trends in hospital readmissions for four high-volume conditions, 2009-2013: statistical brief \#196. Healthcare Cost and Utilization Project (HCUP) Statistical Briefs. Rockville; 2015. 